\title{
Cronología Bibliotecaria Mexicana 1900-1988
}

\author{
Rosa María Fernández de Zamora \\ Investigadora del CUIB
}

\section{RESUMEN}

Eneste trabajose in tentadaraconocercronoló gi camente acon te cimien tos bibliotecariosimpor tan tes suce didosenelsiglo XX y que pueden servir de indicadores del desarrollo histórico de la biblioteca mexicana.

\section{ABSTRAC}

This study has its to de tail chro no li gically the im por tant li brary events which occu rred in the $20^{\text {th }}$ cen tury and which ser ve as indicators of the historical developments in Mexican librarianship.

Se puede definir como cronología a la serie de acontecimientos que se citan por orden de fechas.

En la bibliotecología mexicana, los ensayos cronológicos son prácticamente inexistentes; solo se conoce el artículo de José A. Inclán: "Efemeri des de labibliote ca Nacional", publicado en 1996, en él están registrada las fechas más importantes relacionadas con esta institución.

En el en sa yo que aho ra se pre sen ta se in ten ta dar a co nocer, en un marco cronológico, acontecimientos bibliotecarios importantes que han sucedido en este siglo y que pueden servir de fuen te de re fe ren cia para el análi sis del de sa rrollo histórico de la bibliotecología mexicana. Este trabajo no pretende ser exhaustivo.

Con base en lo anterior la cronología tiene las siguientes características: se citan los principales acontecimientos a par tir de 1900. Se si gue, año con año, el acon te cer bi blio tecario contemplando todos los aspectos posibles: congresos,legislación, fundación de escuelas, seminarios, publicaciones periódicas y publicaciones monográficas singulares como puede ser el caso de la publicación de las series sobre la historia de las bibliotecas mexicanas, caso único en la bibliotecología hispanoamericana, etc. Esto permitiraal lec tor re lacio nar los he chos acon te cidos conel desarrollo bibliotecario a nivel nacional e internacional.

Se tra tó de que la cro no lo gía sólo tu vie ra la des crip ción del hecho citado, sin llegar a analizarlo, ya que no es la finalidad de la misma.

Se es pe ra que la in for ma ción re co pi la da pue de ser de uti lidad, que pueda ser enriquecida en futuras ediciones y que se subsanen las lagunas que se detecten.

1900 se publica: la clasificación decimal de Melvil Dewey para bibliotecas: tablas compendiadas. Tr. Maximiliano M. Chabert. México. Secretaria de Fomento. Traducción delas publicadas porlaoficinaInternacional de Bibliografía de Bruselas.

19004 Decreto del 12 de marzo que contiene el reglamento de la Biblioteca Cepeda, Mérida Yuc., Periódico oficial. 
1904

1905

1912

1914

1915 Creación de la Academía de Bibliografía en Veracruz, Ver., (14 de abril). Se programaron 25 conferencias referentes a la teoría de la clasificación de bibliotecas y archivos.

1916 El24 de junio, se ina gu ra la pri me ra Escuela de Bibliotecarios y Archivistas en la Biblioteca Nacional. Fue cerrada en 1918.

1921 Creación del Departamento de Bibliotecas como uno de los tres Departamentos que conformaron la Se cre ta ría de Edu ca ción Pú blica en sus inicios.

1920-1924 José Vasconcelos, Secretario de Educación Pública, establece cerca de 2000 bibliotecas públicas en todo el país.

1922 Se publica el primer número de El libro y el pueblo. Año 1; Tomo 1; Marzo $1^{\circ}$ revista mensual bibliográfica. Organo del Departamento de Bibliotecas de la SEP.
Reglamento para el prés ta mo de li bros fue ra de labiblio te ca. Se cre taría de Edu ca ción Pública. Departamento de Bibliotecas. (agosto $\left.1^{\circ}\right)$

1924 Se inagura la biblioteca pública Miguel de Cevantes, en el primeredificiocons trui doen México para albergar una biblioteca.

1925 Se inagura la Asociación de Bibliotecarios Mexicanos. Primera de este tipo en el país. Publicó un boletín.

1926

SefundalaEscuelade Bibliotecarios de pendientes del Departamento de Bibliotecas de la SEP. Funcionó aproximadamente dos años.

1925

Se pu bli ca el pri mer nú me ro de la se ries Monografías Bibliografías Mexicanas porlaSecretaría de Relaciones Exteriores, bajo la dirección de Genaro Estrada. En total se publicaron 31 vols., hasta el año de 1935.

1924 Juana Manrrique delara, primerabibliotecaria mexicana que hizo estudios formales de biblioteconomía en el extranjero, egreso de la Library School of New York Public Library.

1925 Requisistos que de benlle nar los as pirantes a bibliotecario.Circular50. SEPDepartamento de Bibliotecas. (30 de diciembre).

1925

Reglamen todelas Bibliote cas Públicas de la Secretaría de Educación Pública. Febrero.

Secele brael se gun do Con gre so Nacional de Bibliotecarios en México, D.F.

Juana Manrrique de Lara inicia en el Departamento de Bibliotecas de la SEP cursos de Biblioteconomía por correspondencia. Termina en 1931.

1929 El 26 de julio la Biblioteca Nacional pasa, por decreto presidencial, a depender de la Universidad Nacional Autónoma de México. 
1934

ReglamentodelaBibliotecaPúblicaCentral del Estado de México. Expedido por el Gobernador en el Diario Oficial del Estado de México.

1936 Se fun da la Biblio te ca del H. Con gre so de la Union. (4 de septiembre)

1937 SecelebraelPrimerCongresoBibliográfico Mexicano.Primer Ateneo Nacional de Ciencias y Arte.

1937 Cursos por correspondencia de Bibliotecono mía y Ar chi vo no mía a car go de Fran cis co Gamoneda. Terminan en 1938.

1940 Se pública el primer número del Boletín Biblio gráfico Mexicano.V.1, No 1, ene ro.Editorial Porrúa.

1942 Se inagura la Biblioteca Benjamin Franklin en Pa seo de la Re for ma 34, el 13 de abril. Inicia sus servicios el día 20. Funcionó muchos años como biblioteca pública.

1943 Se publica el ensayo de una bibliografía de bibliografíamexicanas por Agustín millares Carlo y José Ignacio Mantecón.

1944 Decre to que prohi be la ex por tación de documentosoriginales relacionados con la Historia de Mé xi coy de los li bros que por su ra re za no sean fácilmente sustituibles. Diario Oficial (6 de marzo).

1944 Sellevaacabo Tercer Con gre so Nacionalde Bibliotecarios y Primero de Archiveros. Del 21 al 28 de Octubre. Se propone la apertura de una escuela nacional de bibliotecarios y se pre sen ta un pro yec to de Ley Biblio te ca ria Federal.

1945 Se fun da la Es cue la Na cio nal de Bi blio te carios y Archivistas, ahora Escuela Nacional y Archivonomía (ENBA), en la que se inician los estudios a nivel licenciatura, en esos campos en México. (20 de julio). Primer director: Francisco Orozco Muñoz.
1946 Inaugu ración de labiblio te ca de Méxi co por José Vasconcelos (27 de noviembre). Con la presenciadel presiden te de la Re públicaManuel Ávila Camacho.

1949 Catálogo colectivo de publicaciones periódicas exis ten tes en la Ciu dad de Méxi co, sección de medicina y ciencias biológicas: Biblioteca Benjamín Franklin. Comisión CoordinadoradelaInvestigaciónCientífica.

1950

1956

1956

El 27 de agosto, se crea la Asociación de Bibliotecas y Bibliotecarios de Universidades de Enseñanza Superior de la República Mexicana (ABBUIESRM). En noviembre de 
1965 cambió de nombre: Asociación de Bibliotecarios de Instituciones de Enseñanza Superior y de Investigación (ABIESI) y en diciembre de 1986 se registra como asociación civil. ABIESI, A.C.

Boletín de la Asociación Mexicana de Bibliotecarios. V.I, No. 1, oct/dic 1957- V. 1, nos. 4-5, jul/dic 1958.

Segundas Jornadas Mexicanas de Biblioteconomía, Bi blio gra fía y Can je, San Luis Potosí, (12-15 de abril). Orga ni za das por AMB y ABBUIESRM.

Se restablece el Instituto Bibliográfico Mexicano en la Biblioteca Nacional para que elabore la Bibliografía Nacional de México. ( 31 de mayo).

1960 IIIJornadas MexicanasdeBiblioteconomía. Ciu dad de Mé xi co. (5-8 de di ciem bre) Or ganizadas por AMBAC.

1961 SeminarioLatinoamericanodeBibliografía, Documentación y Canje de Publicaciones (Tercer Seminario Bibliográfico de Centro América y del Caribe). Ciudad de México. (21 nov - 4 de dic). Organizado por la Biblioteca Nacional, Centro de Documentación Científica y Técnica de México y UNESCO.

1962 Anuario de Biblioteconomía y archivonomía. UNAM. Fa cul tad de Fi lo so fía y Le tras. año I, 1961 - año V, 1965.

1963 Directorio de Bibliotecas de la República Mexicana. SEP. Departamento de Bibliotecas.

1964

La Biblioteca Nacional de Antropología e Historia se instaló en el edificio del Museo Nacional de Antropología, en Chapultepec.

Decreto de depósito legal que establece que los editores deben remitir dos ejemplares de sus publicaciones a la Biblioteca Nacional y a la BibliotecadelCongreso.(9 defe bre ro).
IV Jornadas MexicanasdeBiblioteconomía, Jalapa, Ver. (5-8 de mayo) organizadas por AMB AC. Tema: Las bibliotecas en la vida nacional.

1966

Directorio de Bibliotecas de la República Mexicana. 2 ed., SEP. Departamento de Bibliotecas.

Anuario de Bibliotecología y Archivología. UNAM. Fa cul tad de Fi lo so fía y Le tras. Época 2, V. 1, 1971. 
1970

Directorio de Bibliotecas de la República Mexicana 4 ed., SEP. Departamento de Bibliotecas.

1971 Fundación del Centro de Información Científica y Humanística(CICH) de UNAM.

1971 Notas bibliotecológicas. Biblioteca Benjamín Franklin 1971-

1972 Seminario sobre revista cinetíficas nacionales. San Juan del Río, Qro. (28 - 30 de septiembre). CONACYT.

1972 Anuario de Bibliotecología, Archivonomía e informatica. UNAM Facultad de Filosofía y Letras. Epocan 3, año 1, 1972 - año VIII, 1979.

1973 Directorio de Bibliotecas de la República Mexicana. 5 ed. SEP. Departamento de Bibliotecas.

1973

Se iniciaelcursoin ten si vode en tre na mien to para bibliotecarios. Septiembre de 1973. Se imparte el último en 1978. ENBA-CONACYT.

1974

Remedia. V.1. No. 1 Publicación de la Direc ción de Bi blio te cas del IMSS. Dejo de pur blicarse.

1974

Reunión Nacional de Directores y Bibliotecarios de Escuelas y Facultades del Sector Salud. San Luis Potosí, S.L.P., (6-8 de junio).

1974

Jornadas Mexicanas de Biblioteconomía. Gua na juat, Gto. (17-22 de nov.). Or ga ni zadas por AMBAC, Tema: Integración del Servicio Nacional de Bibliotecarios.

1974 I Reunión Nacional de Bibliotecarios y Documentalista Gubernamentales. Acapulco Gro. (18 - 20 de junio). Comité Técnico Consultivo de Centros de Documentación. ABIGMAC.

1974
El estatuto del personal académico de la UniversidadNacional Au tónomadeMéxico posibilitaalaDirección GeneraldeBibliote-

cas a tener personal académico. 28 de junio. Esta disposición influyó posteriormente en otras universidades.

1975

1975

1976

1976 
1976

Catálogo colectivo de publicaciones periódi cas exis ten te en las bi blio te cas de la Re pút blica Mexicana. 2 ed. CONACyT

1976 SeminariosobreEducación y Adies tramiento. Cd. De México. FID/CLA, ALEBCI, UNESCO.

1976 Se ini cia el Ser vi cio de Con sul ta a Ban cos de Información (SECOBI) de CONACyT que conecta a las bibliotecas mexicanas con los principalesbancos deinformación au to matizados internacionales.

1976 Catálogo colectivo de publicaciones periódicas existentes en las bibliotecas de la UNAM. Dirección General de Bibliotecas

1976 El decreto del 6 de noviembre establece como Día Nacional del Libro el 12 de Noviem bre en que se con me mora el na taliciode Sor Juana Inés de la Cruz.

1977 I Semana de Bibliotecología. Guadalajara, Jal. (28 marzo -1 abril). Universidad Autónoma de Guadalajara. Tema: Información para el desarrollo.

1977 VIII Jornadas Mexicanas de Biblioteconomía. Guadalajara, Jalisco ( $1^{\circ}-6$ de mayo). Organizadas por AMBAC. Tema: La proble má ti ca de las bi blio te cas en Mé xi co y sus soluciones.

1977 Calendario de reuniones profesionales en el cam po de bi blio te co lo gía y cien cias de la infor ma ción. No. 1, ju lio - . Cam bió a: Re u niones profesionales de biblioteconomía y ciencia de la información. Calendario semestral. UNAM-DGB.

1977 Cien cia Bi blio te ca ria. V. 1, no.1, sept. - v.7, no. 2, abril 1985. 26 números publicados.

1977 Seminario de ABIESI, Seminario de cooperación en bibliotecas y centros de información. Sal tillo, Coah.(14-16 de sep tiem bre).

1978 Se establece en México el ISBN (Número Internacional Normalizado de Libros). Co- ordinado por la Dirección General del Derecho de Autor de la SEP.

1980

1978

1978

1978

1978

1978

IRESIE. I ndice de Revista de Educación Superior e Investigación. V. 1, No. 1-, UNAM.CentroUniversitariode Tecnología 
Educacional para la Salud (CEUTES) CONACYT.

1979

Seminario de ABIESI, Seminario de Arquitectura de las Bibliotecas, Morelia, Mich. (6-8 de septiembre).

1979

IIISemana de Bibliotecología. Guadalajara, Jal. (24-28 de septiembre). Univerisdad Autónoma de Guadalajara. Relaciones Interbibliotecarias en México.

1979 Directorio de Bibliotecas de la República Mexicana. Ed., SEP. Dirección General de Publicaciones y Bibliotecas.

1979 Crea ción de la Maes tría en Bi blio te co lo gía y Ciencias de la Información en la Universidad de Guanajuato.

1980

Informaciones del Colegio Nacional de Bibliotecarios. V.1, No. 1, enero -

Mesa Redonda sobre formación de recursos humanos para las bibliotecas. Guanajuato, Gto. (18-22 febrero). AMBAC.

1981

XIJornadasMexicanas deBiblioteconomía. México, D.F., (5-9 de mayo). Organizadas por AMBAC. Tema: El usuario.

1980 Boletin InformativoBIBAC. No. 1. Cambió de nombre a Boletín BIBAC

Anuario de Bibliotecología. UNAM. Facultad de Filosofía y Letras. Epoca 4, año 1-

1980 Mesa Redonda ABIESI. Los medios audiovisuales en la biblioteca. Aguascaliente,

1981

1980 Carta Informativa DESIE. Documentatalista en Educación Superior e Investigación Educativa.Año1,No.1-dejódepublicarse.

1980 Informes Técnicos de la DGB/UNAM.1980-

1980

IV SemanadeBibliote cología. Guadalajara, Ags. Cal. (4-6 de septiembre).

II Mesa Redonda sobre Formación de Recursos Hu manos paralas Bibliotecas. Oax tepec, Mor. (2-6 de marzo) AMBAC. Tema: Anteproyecto del núcleo básico de materias para la licenciatura.

XII Joranadas Mexicanas de Biblioteconomía. San Luis Potosí, S.L.P. (4-8 de mayo). Organizadas por AMBAC. Tema: libre.

III Mesa Redonda sobre Formación de Recursos Humanos para las Bibliotecas. Guanajuato Gto. (9-11 de julio). Ciudad de Méxi co (10-12 de sep tiem bre) Co le gio Nacional de Biblio te carios, A.C. Tema: Re cursos humanos nivel maestría.

Se inau gu ra el Edi fi cio de la Biblio te ca de la UAM Azcapozalco (26 de junio).

1981 SefundalaEscuela de Bibliote cología, nivel licenciatura, en la Universidad de Guadalajara.

Primerseminario deCon servación de Documen tos, Li bros y Ma te ria les Gráficos. México, D.F. (24 de agosto). Archivo General de la Nacion, S.R.E., Biblioteca Nacional, Instituto de Estudios y Documentación Históri$\cos$, A.C. Jal. (9 sept.-4 oct.). Universidad Autónoma de Guadalajara. Tema: Cooperación bibliotecaria: estado actual y perspectiva.
VSe mana de Bibliote co logía. Aji jic, Jal.(12 - 16 de octubre). Universidad Autónoma de Guadalajara.TemaAdministraciónbibliotecaria eficiente: Imperativo Nacional. 
1981

1981

1981

1981

1982

1982

1982

1982

1982

1982

1982

1982
Reunión Nacional de Usuarios de SECOBI.

1982

$1^{\text {a }}$ Anual. Se celebra el mes de octubre.

Seminario de ABIESI. Seminario de Automatización 81: Las Bibliotecas en México, D.F. (4-7 de noviembre).

1982

Se establece el Comitá de Conservación de Documentos, Libros y Materiales Gráficos (CODOLMAG). (18 de noviembre)

Creación del Centro Universitario de Investigaciones Bibliotecológica en la UNAM, (CUIB). (14 de diciembre)

Boletín de la Asociación Poblana de Bibliotecarios, No. 1 marzo-

XIII Jornadas Mexicanas de Biblioteconomía. Hermosillo, Son. (3-7 de mayo). Organizada por AMBAC. Tema libre.

Coloquio sobre Investigación Bibliotecológica. Mé xi co, D.F. (28-29 de ju nio). UNAM - CUIB. Tema: Conocer los programas de investiga ciónquese de sarrollan en México.

Primer encuentro de profesores y estudiantes del Colegio de Bibliotecología(16-20de agos to). UNAM. Fa cul tad de Fi lo so fía y Letras.

VISe ma na de Bi blio te co lo gía. Aji jic al. (27 sept- $1^{\circ}$ de oct.) Universidad de Guadalaara. Tema: del arco de la rea li dad al de sa rro llo bibliotecario inmediato.

Pie de página. Revista bibliográfica. Año 1 No. 1 sept. Oct. 1982-. Se pblicaron 12 nos. Dejó de publicarse.

Primer encuentro de Bibliotecarios de la Universidad Nacional Autónoma de México. Ciudad de México. (4-6 de octubre). UNAM Dirección General de Biblioteca. Tema: Prospectiva de biblioteca universitaria.

Informa. No.1- CICH - UNAM.

1982

1983
El Colegio Nacional de Bibliotecario publica:Recomendaciones sobrecapacitación del personal no profesional y lista de acttividades profesionales y no profesionales.

Boletin Informativo de la AMBAC. Nuevo Leon. Año 1, No 1 nov/dic-

Boletín del ISSN. No. 1, nov-CONACYT. Centro Mexicano del ISDS.

Boletín DUP. Disponibilidad Universal de Pblicaciones. IFLA . No. 1 - México, D.F.

XIV Jornadas Mexicana de Biblioteconomía. Zacatecas Zac. (2-6 de mayo) Organizadas por AMBAC. Tema: libre.

Se funda la Asociación Nacional de Bibliotecarios Agropecarios (ANBAGRO).

I Seminario sobre Conservación Restauración de Ma te rial Bi blio gráfi co. Cd. de México. (20-22 de julio). UNAM - CUIB.

Seminario sobre Políticas y Procedimientos de selección en Bibliotecas Mexicanas. Cd. de México. UNAM - CUIB.

Se establece el Programa Nacional de Bibliotecas Públicas 1983 - 1988. (2 de agosto).

IIColoquiodeInvestigación Bibliotecológicas. Cd. de México. (26-27 de septiembre). UNAM - CUIB. Tema: problemas de investigación bibliotecológicas.

1983 I Reunión Nacional de Responsables de los Sistemas Bibliotecarios de las Universidades Públicas Estatales. Campeche, Camp. Universidad del Sudeste. SEP. Dirección GeneraldeInformaciónCientíficay Su peración Académica.

1983 Seminario Nacional sobreCatálogos Colectivos de Publicaciones Periódicas Mexicanas. Cd. de México. (3-7 de octubre). Hemeroteca Nacional. UNESCO. 
1983

1983

1983

1983

1984

1984

1985

1985

1985

1985
Seminario de Publicaciones Periódicas. Cd. de México. (19-21 de octubre). BIBAC.

Segundo Encuentro de Bibliotecas de la UNAM. Cd. de México. (14 - 16 de nov.) Tema: Significado y al cancedelabiblioteca universitaria en la sociedad mexicana.

IV Mesa Redonda sobre Formación de Recur sos Hu ma nos para las Bi blio te cas. Cd. de México. (16 - 18 de noviembre), (8- 9 de marzo) y (7de junio, 24 de septiembre de 1984) Colegio Nacional de Bibliotecarios, A.C., UNAM - CUIB. y Co le gio de Cien cias y Humanidades de la UNAM.

Se mi nario de ABIESI. IISe mi na rio de Cooperación de Bibliotecas y Centros de Información. Monterrey, N.L. (24 - 26 de noviembre).

Segundo Encuentro de profesores y estudiantes deL Colegio de Bibliotecología (5-6 de diciembre)UNAM. Facul tad deFilosofía y Letras.

ANBAGRO informa. V.1 No. 1 ene/dic.

XV Joranadas Mexicanas de Biblioteconomía Tlax ca la Tlax. (30 de abril-4 de mayo). Organizadas por AMBAC. Tema: libre

Boletín bibliográfico y de información. UAM, Iztapalapa. Coordinación de Servicios Documentales. No. 1-4 dejó de publicarse.

VIIISemanadeBibliotecología. Guadalajara, Jal. (25 - 27 de marzo). Universidad Autóno made Guadalajara. Tema: Labiblioteca integradora de las ciencias de la información.

XVI Jornadas Mexicanas de Biblioteconomía. Pa chu ca, Hgo. (6-10 de mayo). Or ga nizadas por AMBAC. Tema: libre.

IV Coloquio sobre Investigación Bibliotecológica. Cd. de México. (29-31 de julio).
UNAM - CUIB. Tema: Métodos y Técnicas de la invetsigación social aplicados a la bibliotecología.

1985 Primer bancomexicanode in for mación disponible a través de SECOBI: SIE-BANXICO (Sistema de Información Económica de México).

1985 I Coloquio sobre Automatización en las Bibliotecas de México. Colima, Col.

1985 Cuar to En cuen tro de alum nos y maes tros del Colegio de Bibliotecología (2-5 de semptiembre). UNAM. Facultad de Filosofía y Letras.

1985 Libros de México. Revista trimestral, Cámara Nacional de la Industria de la Editorial Mexicana. CEPROMEX. No. 1, oct/dic

Se crea el Co mi té por Re cons truc ción de Biblio te cas, pre si di do por la AM BAC. Se ocur pa de las bibliotecas dañadas por los sismos del 19 y 20 de septiembre.

1985

Seminario de ABIESI: Interacción entre la biblioteca y la información; experiencias y orientaciones en el ámbito de la tecnología de la información. San Luis Potosí, S.L.P. (14 -16 de noviembre).

1986

INFOBILA. Se inicia la recopilación de infor ma ción para for mar el Ban co de Da tos de Información Bibliotecologica Latinoamericana. UNAM - CUIB 1986-

1986

III Reunión Nacional de Responsables de los Sistemas Bibliotecarios de las Universidades Públicas Estatales. Cuernavaca Mor. Universidad Autónoima de Morelos. SEP. Dirección General de Información Científica y Superación Académica.

Biblioteca Universitaria. Boletín Informativo de la Dirección General de Bibliotecas. UNAM. V.1, No. 1, ene/mar- 
La AMB AC empieza a recibir donativos de asociaciones extranjeras de bibliotecarios, principalmente de IFLA y ALA, para la restauración de bibliotecas dañadas por los sismos. Febrero.

1986

1986

1986

1986

1986

1986

1986

1986
I ReuniónNacional de Redes de Bibliotecas y Centros de Información. Saltillo, Coah. (12 - 15 de marzo). CONACYT.

1986

IICo lo quio so bre Au to ma ti za ción en las Bibliotecas. Cd. de México. (17 - 18 de abril). UAM- Xochimilco.

XVII Jornadas Mexicanas de Biblioteconomía. Puebla Pue. (28 de abril -2 de mayo) Organizadas por AMBAC. Tema: libre.

Investigación Bibliotecológica. Archivonomía, bibliotecología e información. Revista se mes tral. UNAM - CUIB V. 1, No. 1, agosto.

V Coloquio sobre Investigación Bibliotecologica. Cd. de México. (25 - 26 de agosto). UNAM - CUIB. Tema: Inquietudes y experiencias relacionadas a la investigación bibliotecológica :

Se ini cia la pu bli cac ción de la se rie: His to ria de las bibliotecas en los Estados de la Repúbli ca Me xi ca na con la His to ria de las Bi bliotecas en Veracruz, por Roberto Williams García, Historia de las bibliotecas en Chiapas, por Prudencio Moscoso Pastrana e Historia de las Bibliotecas en Zacatecas, por Ra mi roLafuen te López. SEP. Direc ción General de Bibliotecas.

IXSemanade Bibliote cología. Guadalajara, Jal. (21 - 31 de octubre). Universidad Autónoma de Guadalajara. Tema: Tecnologías relacionadasconalmacenaje, procesamiento y transmisión de información. Nuevo papel delbibliotecario en la profesión, la industria y los servicios.

V Encuentro de estudiantes y profesores de Bibliotecología. (10 - 14 de noviembre).
UNAM. Facultad de Filosofía y Letras. Colegio de Bibliotecología.

1986 Seminario de ABIESI. El impacto de la crisis en las biblio te cas de en se ñan za su periore in ves ti ga ción. Cd de Mé xi co. (12-14 de noviembre).

Fran qui cia pos tal. De cre to del 31 de di ciembre. Se cre ta ría de Ha cien da y Cré di to Pú blico "Art. 147...no pagarán el derecho de correo la correspondencia entre bibliotecas públicas que contengan libros, así como envíos de libros que en forma gratuita a éstos, hagan las empresas editoriales residentes en México". Franquicias gestionadas por ABIESI.

1986 Se inicia la publicación de la serie: Historia de las Biblio te cas en Mé xi co, con el V. 1, Las Bibliotecas Novohis panas, por Ig nacio Osorio. SEP. Dirección General de Bibliotecas.

Circular 1-51-187 del Ser vi cio pos tal Mé xicano relacionada con la franquicia postal para las bibliotecas públicas. (2 de enero).

1987 Cuadernos de ANBAGRO. No. 1-

1987 Decreto Presidencial que consolida la Red Nacional de Bibliotecas Públicas (RENABIP) (8 de enero).

1987 Boletín CENIDS. Bo le tín del Cen tro Nacional de Información y Documentación en Salud. Se cre ta ría de Sa lud. V. 1, No. 1, ene/feb.

1987 Diseminación selectiva de información de CENIDS. No. 1- En 1986 un número especial.

1987 XVIIIJornadas Mexicanas deBiblioteconomía. Cd. de Mé xi co. (2-6 de mar zo). Or ga nizadas por AMBAC. Tema: Hacia el siglo XXI:Lasbibliotecas mexicanas, evaluación y proyección.

BoletíninformativodelColegiodeBiblioteco lo gía. UNAM. Fa cul tad de Fi lo so fía y Letras. No. 1, junio- 
1987

PrimerseminariosobrePolíticas Nacionales de Información para la Invetsigación y el Desarrollo.(24-25 de agos to). CONACYT.

1987

VI Coloquio sobre Invetsigación Bibliotecológica. Cd. de México. (3-4 de septiembre). UNAM - CUIB. Tema: 1. Formación de lectores. 2. Clasificación en las bibliotecas mexicanas. Necesidad del usuario o fastuosidad del bibliotecólogo. 3. Normalización y problemas de frontera de investigación.

1987 X Semana de Bibliotecología. Guadalajara, Jal.(9-11 septiem bre). Uni ver si dad Autónoma de Guadalajara. Tema: Usuario de la información.

1987 Reunión de trabajo sobre cooperación bibliotecaria. Cd. de México. (17 - 18 de septiembre). ITAM, SEP, ANUIES, Consejo Británico.

1987 Se reinaugura la Biblioteca Pública Pavón, restaurada de los daños sufridos por los sismos de 1985 con los donativos que asociacionesextranjeras debibliotecariosenviaron a la AMBAC.

1987 Decreto por el que se aprueba el Programa Integral de Fomento a la Industria y Comercio del Libro, Diario Oficial 2 de octubre.

1987 VI Encuentro de bibliotecarios de la UNAM. Cd. de México. (5-7 de octubre). Tema: Los re cur sos hu ma nos en las bi blio tecas de la UNAM: Formación profesional y capacitación.

1987

IV Reu nión Na cional de Res pon sable de los Sistemas Bibliotecarios de las Universidades Públicas Estatales. Villahermosa, Tab. (4-6 noviembre). Universidad Juárez Autónoma de Tabasco. SEP. Dirección General de Información Cietífica y Superación Académica.
Tercer Coloquio sobre Automatización en Bibliotecas, Colima, Col. (18 - 19 de noviembre). Universidad de Colima, CONACYT, SEP. Tema: Experiencias institucionales en materia de automatización. Avances tecnológicos. Alternativas de cooperación interintitucional.

Seminario de ABIESI. Economía de la infor ma ción. Gua da la ja rA, Jal. (28 - 30 de noviembre).

VI Encuentro de alumnos y maestros de Bibliotecología. México, D.F., (2 - 4 de diciembre) UNAM. Facultad de Filosofía y Letras. Colegio de Bibliotecología.

Ley General de Bibliotecas. En los primeros días de diciembre de 1987 la Cámara de Senadores aprueba la Ley General de Bibliotecas enviada por el Ejecutivo. El 17 del mismo mes la Cámara de Diputados la aprueba sin modificación alguna. Esta Ley contempla la integración de un Sistema Nacional de Bibliotecas. El Presidente expide el decreto correspondeinte el 21 de diciembre.

Se publica la Ley General de Bibliotecas en el Diario Oficial del jueves 21 de enero, págs. 14- 16.

XIX Jornadas Mexicanas de Biblioteconomía. Villahermosa Tab. (7-9 de marzo). Organizadas por AMBAC. Tema libre.

Rea nu dadción to tal de los ser vi cios de la Biblioteca Benjamín Franklin en su domicilio Londres 16, después de haber sido cerrada por los daños sufrido durante los terremotos de 1985. (13 de abril).

1988 Elsecretariode Educación Públicainaugu ra la Biblioteca del Acervo Editorial de la SEP en Argentina 12, Centro. (20 de abril). 